\title{
RNA interference does not function as an innate antiviral response in mammalian somatic cells
}

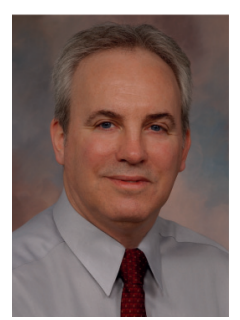

"... it seems safe to conclude that RNAi evolved very early in eukaryotic evolution as an antiviral defense mechanism and that it continues to play this role in plants and invertebrates."

Bryan R Cullen

Department of Molecular Genetics \& Microbiology \& the Center for Virology, Duke University, Durham, NC USA = bryan.cullen@duke.edu

RNA interference (RNAi) is a sequence-specific, post-transcriptional mechanism of gene inhibition, triggered by long dsRNAs, that plays a key role in the innate antiviral immune response in plants and invertebrate animals. Surprisingly, it has now become increasingly apparent that RNAi does not function as an innate immune response in mammalian somatic cells, which have lost the ability to effectively process dsRNAs into the siRNAs that mediate RNAi. Remarkably, the RNAi system is active in mammalian germ cells, where it likely functions to inhibit retrotransposon mobility. This suggests that evolution has selected for the tissue-specific loss in mammals of an innate antiviral mechanism that is clearly protective in many non-mammalian species.

"Surprisingly, it has now become increasingly apparent that RNAi does not function as an innate immune response in mammalian somatic cells, which have lost the ability to effectively process dsRNAs into the siRNAs that mediate RNAi."

RNAi, as originally described [1] and as defined here, is a process whereby the introduction into cells of a long, dsRNA segment derived from a cDNA copy of an mRNA results in the degradation of that mRNA and, hence, inhibition of expression of the cognate gene. Subsequent work revealed that the introduced dsRNA is cleaved into approximately 22-bp dsRNA segments by the action of the cytoplasmic RNase III enzyme Dicer. One strand of this 22-bp dsRNA, referred to as a 'small interfering RNA' (or siRNA), is then incorporated into the RNA-induced silencing complex, the key component of which is a member of the cellular Argonaute (Ago) protein family. The siRNA guides RNA-induced silencing complex to cognate mRNA species which are then cleaved at the site of homology and degraded. Key characteristics of siRNAs include their approximately 22-nucleotide size and the fact that they derive equally from both strands of introduced dsRNAs; in other words, they are equally antisense - and therefore able to induce cleavage of the cognate mRNA - and sense, in which case no fully complementary mRNA target may exist.

Consideration of the selective pressures that led to the evolution of RNAi suggests that RNAi originally arose as an antiviral innate immune response. Eukaryotic cells produce very few segments of perfectly duplex dsRNA of $>20$ bp or so, while almost all viruses produce long segments of perfect dsRNA during their replication cycle. These long $\mathrm{d}$ sRNAs arise from the replication of the RNA genome of RNA viruses, all of which - with the exception of retroviruses - must copy their entire RNA genome using a virally encoded RNA-dependent RNA polymerase. Long dsRNAs are also produced in cells infected by many dsDNA viruses due to convergent transcription of highly compact viral DNA genomes. Therefore, long dsRNAs function as a pathogen-associated molecular pattern that alerts the cell to the fact that viral infection has occurred. Moreover, in the case of RNAi, these same long dsRNAs also serve as the substrate for the derivation of siRNAs that can then be used to specifically degrade viral RNA transcripts.

Analysis of cells of plant or invertebrate origin infected with viruses (reviewed in [2]) has in fact

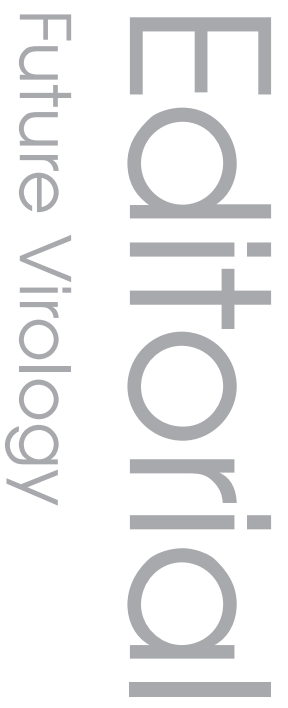

\section{Keywords}

- dsRNA = innate immunity

- RNA interference $=$ siRNA

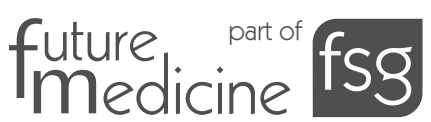


demonstrated that these cells produce high levels of siRNAs of viral origin. Indeed, among the first siRNAs to be reported were those induced in plants upon infection with potato virus $\mathrm{X}$ [3], and virus-derived siRNAs are often the most prevalent small RNAs in virus-infected cells [2]. Moreover, it is now apparent that RNAi is a key component of the innate antiviral immune response in plants and invertebrate animals, as mutational inactivation of the RNAi response leads to hypersensitivity to viral infection and a significant increase in pathogenesis. Moreover, and as expected if RNAi can limit viral replication in vivo, many plant and insect viruses express one or more factors that inhibit aspects of the RNAi response, and the mutational inactivation of these viral gene products results in viruses that are highly attenuated [2]. Therefore, it seems safe to conclude that RNAi evolved very early in eukaryotic evolution as an antiviral defense mechanism and that it continues to play this role in plants and invertebrates.

\section{“...the evidence reviewed so far strongly suggests that mammalian somatic cells are unable to generate siRNAs from long dsRNAs, including those generated during viral infection."}

Based on these data, it was initially thought that RNAi would also serve as an innate antiviral response in mammals. However, it now seems clear that this is not the case. Deep sequencing of small RNA populations from cells infected with a range of RNA and DNA viruses has not produced evidence indicating that mammalian cells share the ability of plant and invertebrate cells to produce functional siRNAs. Specifically, although small RNAs of viral origin are often readily detectable in virusinfected cells, especially those undergoing virusinduced cytopathic effects, they are not clustered at the approximately 22-nucleotide size expected for siRNAs but rather are mostly of random size [4]. Moreover, the ratio of these small RNAs is often strongly skewed towards the more highly expressed strand of the RNA virus, not equally distributed to both strands, as one would expect for siRNAs, although some exceptions have been reported [5]. Finally, and most importantly, efforts to demonstrate that RNAi plays a role in limiting virus replication by knocking down key components of the RNAi response, such as Dicer or Ago2, have not yet revealed such a role for RNAi in mammalian cells [6], even though comparable experiments in invertebrates clearly demonstrate this effect [2].

If RNAi does play a role in inhibiting virus replication in mammals then, by analogy to plant and insect viruses, one would predict that mammalian viruses would have also evolved proteins that inhibit RNAi function. Indeed, a number of reports to this effect have appeared, including papers arguing that the HIV-1 Tat and influenza virus NS1 proteins can block siRNA production and RNAi [7-10]. In the case of HIV-1, this would be an odd result as retroviruses, uniquely among RNA viruses, do not generate long dsRNAs at any stage in their lifecycle. Therefore, there is no obvious viral target for Dicer in HIV-1 infected cells, and even if the coding strand of HIV-1 were to be cleaved by Dicer to generate an siRNA, there is no viral target for it to act on. In fact, careful analysis has now clearly revealed that the claimed ability of HIV-1 Tat to inhibit RNAi likely represents an overexpression artifact in cultured cells [11].

A more interesting example arises in the case of influenza NS1, which was clearly shown to inhibit RNAi in plant and insect cells when ectopically expressed [8-10]. NS1 is a dsRNA-binding protein that functions as an inhibitor of innate antiviral immune responses, especially interferon responses, and influenza viruses lacking NS1 are highly attenuated in wild-type cells. Strikingly, however, NS1 is largely dispensable for influenza virus replication in interferon-deficient cells [12], thus suggesting that inhibition of RNAi by NS1, if it does occur, may not play a significant role. Moreover, ectopic expression of dsRNA binding proteins of bacterial origin in plants also blocks RNAi [13], while expression of an NS1 deletion mutant retaining only the approximately 82-amino acid dsRNA binding domain of NS1 also inhibits RNAi effectively [10]. Finally, influenza virus does not give rise to detectable siRNAs in infected cells [4]. Together, these data argue that NS1 does not function as an inhibitor of antiviral RNAi responses in influenza virus-infected cells.

While it is extremely difficult to prove a negative, the evidence reviewed so far strongly suggests that mammalian somatic cells are unable to generate siRNAs from long dsRNAs, including those generated during viral infection. A recent paper that goes a long way towards proving this point in an in vivo setting examined whether dsRNAs expressed in a transgenic mouse could give rise to functional siRNAs in germ cells and somatic cells in vivo [14]. The context of this research was data from several groups 
demonstrating that long dsRNAs can give rise to functional siRNAs in oocytes and embryonic stem cells $[15,16]$. By contrast, in somatic cells, long dsRNAs activate protein kinase $\mathrm{R}$ (PKR) and induce the interferon system, which results in a global inhibition of protein translation, mRNA destabilization and eventually programmed cell death. Unlike somatic cells, germ cells, especially oocytes, appear to be deficient in PKR function and are not competent to mount an interferon response [17]. Therefore, while it seemed clear that mammalian oocytes are competent for RNAi, it could be argued that detection of long dsRNA-induced RNAi in somatic cells was occluded by a nonspecific PKR- and interferon-driven response. To address this issue, Nejepinska and coworkers expressed a dsRNA under the control of a constitutive, ubiquitously active promoter in mice [14]. This dsRNA, while readily detectable, nevertheless did not induce PKR or interferon responses, or induce developmental defects. Analysis of small RNAs from different tissues in the mice revealed that siRNAs were not effectively produced in somatic tissues, such as brain and kidney, that expressed this dsRNA. While there were too few oocytes available to perform deep sequencing on this tissue, introduction of a green fluorescent protein-based indicator construct containing a segment complementary to the expressed dsRNA allowed these authors to show that functional siRNAs are produced in oocytes but not in other tissues. Together, these experiments reveal that a dsRNA that is fully competent to be processed into active siRNAs in mammalian germ cells is not competent to be processed into siRNAs in somatic tissues in the same animal.

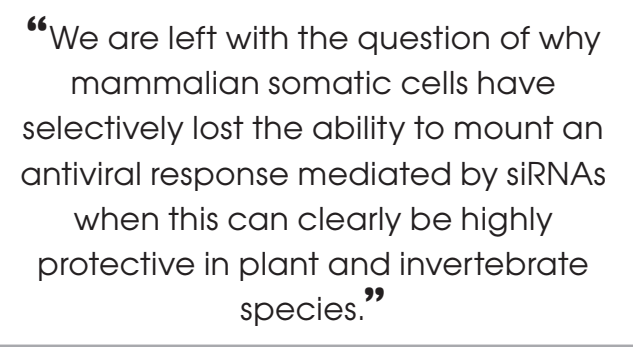

These studies raise several important questions, including: why are mammalian germ cells competent for RNAi while somatic cells are not? It is likely that RNAi in germ cells functions to inhibit the mobility of endogenous retrotransposons such as LINEs, which are transcribed at high levels in germ cells. Because there are $>10^{5}$ copies of LINE1, for example, in the human genome that are inserted into many genes in both orientations, dsRNAs of LINE1 origin are undoubtedly produced in germ cells, such as oocytes, and can serve as substrates for Dicer cleavage. Retrotransposon mobility is a major potential problem in germ cells, because deleterious retrotransposition events could become fixed in the genome of any offspring. However, retrotransposition is a lesser concern in somatic cells, which represent a dead-end for the newly inserted retrotransposon. The ability to mount an RNAi response may therefore be particularly important in germ cells. Why mammalian somatic cells lack the ability to produce functional siRNAs from long dsRNAs is unclear. Somatic cells express fully functional microRNAs and therefore obviously contain an active Dicer enzyme. One possibility is that mammalian germ cells express a Dicer co-factor that allows Dicer to function as a dsRNAspecific endonuclease, while Dicer appears to act exclusively as a dsRNA-specific exonuclease in somatic cells. It certainly seems that dsRNAs that are flanked by ssRNA sequences or that bear ends occluded by proteins are not susceptible to Dicer cleavage in somatic cells, but are apparently cleaved by Dicer when expressed in germ cells [14].

We are left with the question of why mammalian somatic cells have selectively lost the ability to mount an antiviral response mediated by siRNAs when this can clearly be highly protective in plant and invertebrate species [2]. In part, this no doubt reflects the fact that the dsRNA pathogen-associated molecular pattern can give rise to an effective innate antiviral response in mammals mediated by the interferon system, using detectors of dsRNAs such as PKR and Toll-like receptors. However, it still seems odd that the RNAi-based innate antiviral response that was obviously present and active early in animal evolution has not been maintained. Possibly the analysis of RNAi responses in primitive vertebrate species, or in more complex invertebrate species, might provide a clue to this conundrum.

Financial \& competing interests disclosure

The author has no relevant affiliations or financial involvement with any organization or entity with a financial interest in or financial conflict with the subject matter or materials discussed in the manuscript. This includes employment, consultancies, honoraria, stock ownership or options, expert testimony, grants or patents received or pending, or royalties.

No writing assistance was utilized in the production of this manuscript. 


\section{References}

1. Fire A, Xu S, Montgomery MK, Kostas SA, Driver SE, Mello CC. Potent and specific genetic interference by double-stranded RNA in Caenorhabditis elegans. Nature 391, 806-811 (1998).

2. Ding SW, Voinnet O. Antiviral immunity directed by small RNAs. Cell 130(3), 413-426 (2007).

3. Hamilton AJ, Baulcombe DC. A species of small antisense RNA in posttranscriptional gene silencing in plants. Science 286(5441), 950-952 (1999).

4. Umbach JL, Yen HL, Poon LL, Cullen BR. Influenza A virus expresses high levels of an unusual class of small viral leader RNAs in infected cells. mBio 1(4), E00204-E00210 (2010).

5. Parameswaran P, Sklan E, Wilkins C et al. Six RNA viruses and forty-one hosts: viral small RNAs and modulation of small RNA repertoires in vertebrate and invertebrate systems. PLoS Pathog. 6(2), E1000764 (2010).

6. Shapiro JS, Varble A, Pham AM, Tenoever BR. Noncanonical cytoplasmic processing of viral microRNAs. RNA 16(11), 2068-2074 (2010).
7. Bennasser Y, Le SY, Benkirane M, Jeang KT. Evidence that HIV-1 encodes an siRNA and a suppressor of RNA silencing. Immunity 22(5), 607-619 (2005).

8. Delgadillo MO, Saenz P, Salvador B, Garcia JA, Simon-Mateo C. Human influenza virus NS1 protein enhances viral pathogenicity and acts as an RNA silencing suppressor in plants. J. Gen. Virol. 85(Pt 4), 993-999 (2004).

9. Bucher E, Hemmes H, De Haan P, Goldbach R, Prins M. The influenza A virus NS1 protein binds small interfering RNAs and suppresses RNA silencing in plants. J. Gen. Virol. 85(Pt 4), 983-991 (2004).

10. Li WX, Li H, Lu R et al. Interferon antagonist proteins of influenza and vaccinia viruses are suppressors of RNA silencing. Proc. Natl Acad. Sci. USA 101(5), 1350-1355 (2004).

11. Sanghvi VR, Steel LF. A re-examination of global suppression of RNA interference by HIV-1. PLoS One 6(2), E17246 (2011).

12. Garcia-Sastre A, Egorov A, Matassov D et al. Influenza A virus lacking the NS1 gene replicates in interferon-deficient systems. Virology 252(2), 324-330 (1998).
13. Lichner Z, Silhavy D, Burgyan J. Doublestranded RNA-binding proteins could suppress RNA interference-mediated antiviral defences. J. Gen. Virol. 84(Pt 4), 975-980 (2003).

14. Nejepinska J, Malik R, Filkowski J, Flemr M, Filipowicz W, Svoboda P. dsRNA expression in the mouse elicits RNAi in oocytes and low adenosine deamination in somatic cells. Nucleic Acids Res. doi:10.1093/nar/gkr702 (2011) (Epub ahead of print).

15. Yang S, Tutton S, Pierce E, Yoon K. Specific double-stranded RNA interference in undifferentiated mouse embryonic stem cells. Mol. Cell. Biol. 21(22), 7807-7816 (2001).

16. Wianny F, Zernicka-Goetz M. Specific interference with gene function by doublestranded RNA in early mouse development. Nat. Cell Biol. 2(2), 70-75 (2000).

17. Stein P, Zeng F, Pan H, Schultz RM. Absence of non-specific effects of RNA interference triggered by long double-stranded RNA in mouse oocytes. Dev. Biol. 286(2), 464-471 (2005). 